\title{
Prenatal cystic fibrosis carrier testing: designing an information leaflet to meet the specific needs of the target population
}

\author{
ME Mennie, W A Liston, D J H Brock
}

\begin{abstract}
A questionnaire was given to 180 patients in an antenatal clinic, who were eligible to enter a pilot trial of cystic fibrosis (CF) carrier testing, seeking their views on the information leaflet inviting them to participate; 161 patients $(89 \%)$ entered the trial and 145 patients $(81 \%)$ responded to the questionnaire, including 10 who did not enter the trial. Ninety-six percent of these found the leaflet easy to understand, and $97 \%$ of those partners who read the leaflet also found it easy to understand. Fifteen percent of patients thought the leaflet should give additional information. Most (92\%) had heard of CF before reading the leaflet, television being the most common source of information. Although avoiding the birth of a child with CF was the reason most patients gave for wishing to be screened, almost as many were interested to know their carrier status. The decision to accept or decline testing was taken in conjunction with their partner by $63 \%$ of women. Of those who were screened, $59 \%$ stated that taking the test made them feel reassured, while $38 \%$ felt slightly apprehensive. It was concluded that, with a number of minor amendments, the leaflet met the specific needs of the target patient population.
\end{abstract}

The cloning of the cystic fibrosis $(\mathrm{CF})$ gene in 1989 and the subsequent description of a number of mutant CF alleles has made it possible to detect $\mathrm{CF}$ carriers in the general population. A survey of Scottish CF patients and their parents shows that about $85 \%$ of carriers can be identified by testing for just four mutations. ${ }^{1}$ Although this is less than ideal for a screening test, it has been argued that the severity of the disorder demands an immediate start to pilot trials of carrier detection. ${ }^{2}$ Accordingly we have initiated such a trial in selected antenatal clinics of the major Edinburgh maternity hospital.

Pregnant women are offered the chance of joining the trial by means of a leaflet sent with their antenatal booking clinic appointment. The leaflet emphasises the incomplete nature of the test and that it cannot guarantee against the birth of a CF child. Volunteer women are screened and if found to carry a CF allele their partners are also screened. Couples where both partners are found to be carriers are offered prenatal diagnosis.

A prerequisite to an informed decision to undergo any screening or diagnostic test is knowledge of the test. ${ }^{3}$ Indeed, some argue that the effectiveness of such a screening programme should be assessed by whether the participants have become fully informed. ${ }^{4}$ However, to be effective and useful, educational materials must meet the specific needs of the target population. ${ }^{5}$

The use of a questionnaire to assess patients' attitudes to information leaflets has been described. ${ }^{67}$ Before committing ourselves to a final version of the information leaflet designed for this trial, we tested the acceptability and usefulness of a preliminary version on our target population, by means of a self administered questionnaire.

\section{Subjects and methods}

A pilot trial was run in one antenatal booking clinic for a period of 14 weeks. Along with their booking clinic appointment, patients received a copy of the information leaflet outlining the purpose and process of prenatal CF carrier screening (table 1). The leaflet also incorporated a consent form to be signed by patients entering the screening trial.

A total of 200 antenatal patients attended the booking clinic during the pilot trial. Twenty of these patients were not eligible to enter the screening trial for reasons of late gestation (greater than 18 weeks), abnormality of pregnancy (for example, blighted ovum), or unavailability of partner. Questionnaires were not issued to this group. The remaining 180 antenatal patients were given a questionnaire and a stamped addressed envelope at the booking clinic and asked to complete it at home and return it. Patients were not asked to identify themselves. A total of $145(81 \%)$ returned their questionnaire. Of the 161 patients who entered the trial, 135 responded. Of the 19 patients declining to enter, 10 responded.

\section{Results}

PATIENTS' PREVIOUS KNOWLEDGE OF CF AND SOURCE OF INFORMATION

The questions were:

(1) Had you heard of the disease cystic fibrosis before you read the leaflet?
Correspondence to Dr Mennie.

Received 28 May 1991. Revised version accepted 6 September 1991. 
Table 1 The information leaflet.

Cystic fibrosis carrier testing
Prenatal screening trial

Cystic fibrosis is serious. The average life expectancy is 19 years. Children with cystic fibrosis have repeated chest infections and are usually underweight and undersize. They may need daily physiotherapy and large numbers of tablets to assist digestion. If they live long enough they will probably need a heart and lung transplant.

We think that a woman who is having a baby will want to know the answers to three important questions:

(1) Am I a carrier?

(2) Is my partner a carrier?

(3) Will my baby be affected?

We are running a trial which tests whether or not you are a carrier of a single cystic fibrosis gene. If you are not there is no carrier of a single cystic If you are a carrier it is possible to tell if your husband or

Only if both of you are carriers will you have a 1 in 4 chance of
antion

Only if both of you are
having an affected child.

If you and your partner are both carriers, a prenatal test can tell whether or not your baby will be affected.

$$
\text { Prenatal diagnosis }
$$

This is one option available to couples who have a 1 in 4 chance of having a child with cystic fibrosis. It is possible to diagnose cystic fibrosis in the fetus. If the prenatal diagnostic test shows that the unborn child is going to have cystic fibrosis, you may wish to have an abortion.

Prenatal diagnosis is not recommended for those couples who find abortion unacceptable.

\section{The cystic fibrosis carrier screening test}

A small sample of the blood taken for your other antenatal tests will be used to test if you are a carrier. You will be contacted only if the test shows you are a carrier. only if the test shows you are a carrier.
Testing for the cystic fibrosis gene is still only possible in
$85 \%$ of cases. This means we cannot guarantee that you will not have a child with cystic fibrosis. However, if the test is negative it will greatly reduce that risk.

\section{Testing your partner}

If you are found to carry a single cystic fibrosis gene we would If you are found to carry a sing

A small mouth wash sample will be used to test your partner, so he will not have to give a blood sample.

sample.

If your partner's test is negative then your risk of having a baby with cystic fibrosis is low.

A special leaflet and genetic counselling is available for all couples where one partner has a positive carrier test.

Couples who are both carriers of a single cystic fibrosis gene Genetic counselling is available to couples where both partners are found to be carriers.

The various options open to you will be fully explained. You will have time to think through these options and to discuss them at length with the counsellor.

Further information and advice about prenatal carrier testing for cystic fibrosis

There will be time at the booking clinic to ask any questions, or discuss any concerns you may have about prenatal carrier testing for cystic fibrosis. Counselling is available at any stage of the screening trial. There will be a nurse counsellor available at the antenatal booking clinic.

Prenatal carrier testing for cystic fibrosis is voluntary

Prenatal carrier testing for cystic fibrosis is entirely voluntary.

If you would like to be tested please complete the enclosed form and bring it with you to the antenatal booking clinic.

Important points to remember about prenatal carrier testing for cystic fibrosis

(1) Carrying a single cystic fibrosis gene is completely harm-

(2) Only if both partners carry a single cystic fibrosis gene will there be a 1 in 4 chance that their child will have cystic fibrosis.

(3) Testing for the cystic fibrosis gene is still only possible in $85 \%$ of cases.

(4) Genetic counselling is available for all couples where both partners are found to carry a single cystic fibrosis gene.

5) The prenatal cystic fibrosis carrier screening trial is entirely voluntary.

(6) Remember there are trained staff to give you information and advice - please do ask.

(2) If you had heard of cystic fibrosis, where did you hear about it? (Tick as many options as required.)
There were 145 responses, with 134 (92\%) saying yes. Major sources of information were TV, women's journals, and charity appeal.

PATIENTS' ASSESSMENT OF THE LEAFLET AND UNDERSTANDING OF THE PURPOSE OF CF CARRIER TESTING

The questions were:

(3) In your opinion, is the leaflet introducing the cystic fibrosis carrier screening test difficult or easy to understand?

(4) How many times did you read the leaflet before you felt you understood it?

(5) Do you feel you understand the purpose of cystic fibrosis carrier testing?

(6) Do you feel the leaflet should give more information?

(7) Has your partner read the leaflet?

(8) If your partner read the leaflet, was it, in his opinion, difficult or easy to understand?

(9) Did you make the decision to accept or refuse the screening test alone or with your partner?

The answers of the 145 respondents are listed in table 2 .

PATIENTS' REASONS FOR BEING SCREENED (NUMBERS BASED ON THOSE 135 RESPONDENTS WHO ENTERED THE SCREENING TRIAL)

The question asked was:

(10) If you have decided to take the screening test, is this because (tick as many options as required)

(a) I am interested to know if I carry a single cystic fibrosis gene. (96 respondents)

(b) I do not want my child to have cystic fibrosis. (102 respondents)

(c) My partner wanted me to have the screening test. (22 respondents)

(d) Other (please state reason). (10 respondents)

PATIENTS' REASONS FOR NOT BEING SCREENED (NUMBERS BASED ON THE 10 RESPONDENTS WHO DID NOT ENTER THE TRIAL)

The question asked was:

(11) If you have decided not to take the screening test is this because (tick as many options as required)

(a) I would prefer not to know if I carry a single cystic fibrosis gene. (No respondents)

(b) I am against termination of pregnancy. (10 respondents)

(c) My partner did not want me to take the screening test.

(3 respondents)

(d) Other (please state reason). (No respondents) 
Table 2 Patients' assessment of information leaflet and their understanding of CF carrier testing: numbers (and percentages).

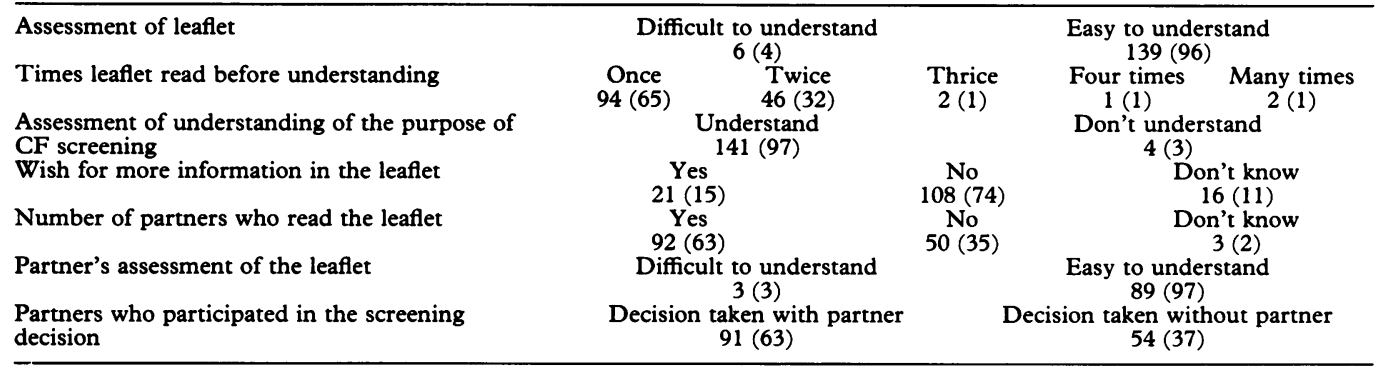

PERSONS WITH WHOM PATIENTS DISCUSSED THE SCREENING TEST (NUMBERS BASED ON RESPONDENTS WHO ENTERED THE TRIAL)

The question asked was:

(12) Have you discussed taking the screening test with anyone? (Tick as many options as required)
(a) Partner
(b) Relative
(c) Friend
(d) GP
(e) Health visitor
(f) Other (state who) (no respondents)
(112 respondents)
(18 respondents)
(21 respondents)
(5 respondents)
(1 respondent)

PATIENTS' ESTIMATION OF THEIR LIKELIHOOD OF BEING A CF CARRIER AND ATTITUDE TO BEING TESTED (NUMBERS BASED ON THOSE 135 PATIENTS WHO ENTERED THE SCREENING TRIAL) The questions were:

(13) How likely do you think it is that you will carry a single cystic fibrosis gene?

(14) How does the thought of the cystic fibrosis carrier test make you feel?

Four options were listed (table 3).

\section{Discussion}

The cystic fibrosis pilot trial began in the antenatal clinic of the Simpson Memorial Maternity Pavilion in October 1990. Since the first programmes of maternal serum AFP screening were developed and started in Edinburgh in 1975, we already had in place antenatal staff broadly trained in the process of explaining the concepts of screening.

With their antenatal booking clinic appointment, patients received a $\mathrm{CF}$ prescreening information leaflet incorporating a consent form. The midwife carrying out the booking procedure ensured that her patient had received a leaflet, had read it (or been able to read it), and was fully aware of the consequences of $\mathrm{CF}$ carrier screening. A genetic nurse was on duty at the clinic to answer patient or staff questions and to offer counselling and one to one sessions, using visual aids, to patients and couples with difficulty in reading or understanding the leaflet. Patients wishing to enter the trial signed a consent form.
Information given before prenatal genetic screening must be adapted to a woman's circumstances and be sufficient to enable her to reach a fully informed decision. ${ }^{8}$ Only too frequently, when educational protocols are being developed, the target population reaction is not sought. ${ }^{9}$ For information and education to be effective, this group needs to be consulted to determine what they need to know or learn. This study attempts to consult antenatal patients about a leaflet designed to inform them about prenatal CF carrier testing.

The response to the questionnaire was high $(81 \%)$. All questionnaires were completed in full and many respondents commented at length on the questions. Most (92\%) claimed they had heard of CF before they read the information leaflet, and many were able to cite the source of their knowledge. This probably reflects the high profile of the Cystic Fibrosis Research Trust in the UK and may not be applicable to other European countries.

There was no correlation between absence of previous information and difficulty understanding the leaflet. Of the six respondents who had difficulty understanding the leaflet (table 2), five had previously heard of CF. However, of the four women who felt they did not understand the purpose of CF screening (table 2), three stated they had not previously heard of CF. Comments indicated that many of this antenatal patient population were also familiar with one major clinical feature of CF, namely lung disease. A concern raised regarding the initiating of CF carrier screening programmes is that only a small percentage of the population are at present familiar with the disease. ${ }^{4}$ This study did not attempt to test subjects' knowledge of CF and it would be misleading to suggest that patients had detailed knowledge. Indeed, more details about the disease itself were requested by all $15 \%$ of respondents (table 2 ) who felt that the leaflet should give more information. Nevertheless, it is encouraging that so many women were previously aware of the disorder.

The Council of Europe, in their official statement on genetic screening, note the role that the media can play in informing and educating the public and recommend they be

Table 3 Patients' estimation of their likelihood of being a CF carrier and their attitude to being screened: numbers (and percentages).

\begin{tabular}{|c|c|c|c|c|}
\hline $\begin{array}{l}\text { Estimated likelihood of being a CF carrier } \\
\text { Antenatal patient's attitude to being screened }\end{array}$ & $\begin{array}{c}\text { Highly likely } \\
1(1) \\
\text { Anxious } \\
4(3)\end{array}$ & $\begin{array}{c}\text { Not very likely } \\
62(46) \\
\text { Slightly } \\
\text { apprehensive } \\
51(38)\end{array}$ & $\begin{array}{c}\text { Most unlikely } \\
72(53) \\
\text { Reassured } \\
79(59)\end{array}$ & $\begin{array}{c}\text { Don't know } \\
0 \\
\text { Don't know } \\
1(1)\end{array}$ \\
\hline
\end{tabular}


kept informed of all aspects of antenatal genetic screening. ${ }^{8}$ In this study, television was the leading source of information about CF. A considerable number of respondents were acquainted with the disease through women's journals and charity appeals. Knowledge of CF was acquired by a number of women through their occupation and in three cases by virtue of having a distant family history of the disorder.

The majority (96\%) of antenatal patients stated that they found the leaflet easy to understand (table 3). There was no correlation between difficulty understanding the leaflet, or failing to understand the purpose of CF screening, and declining the test. All $10 \mathrm{re}-$ spondents who declined the test found the leaflet easy to understand and stated that they felt they understood the purpose of CF screening. One woman took the CF test, despite finding the leaflet difficult to understand and not understanding the purpose of CF screening.

There is a danger in designing an information leaflet that one may make it either too simple or too complex. Many women are confident and articulate users of the antenatal care system and can cope with detailed information. Others lack the basic knowledge to know the right questions to ask. Our solution has been to design a comparatively simple leaflet and to train midwives to pick up those cases who are unsure about what is being offered. A genetic nurse is on standby to give one to one counselling and answer the more sophisticated questions.

Most women $(65 \%)$ stated they had only read the leaflet once before feeling they understood it (table 2). Antenatal bookers receive a considerable volume of material to read along with their clinic appointment and may find they have limited time to read and absorb it all. Those who enter the CF screening trial must sign a consent form and in addition the midwife responsible for booking a patient is instructed to do his or her utmost to ensure that a participant understands the purpose of the screening test. Nevertheless, studies do indicate that a lack of knowledge and understanding of women participating in prenatal screening programmes is an area for concern ${ }^{10}$ and researchers have attempted to design instruments whereby knowledge of such tests can be measured. ${ }^{3} \mathrm{CF}$ carrier screening may carry more obscure risks and considerations than other antenatal screening tests, notably

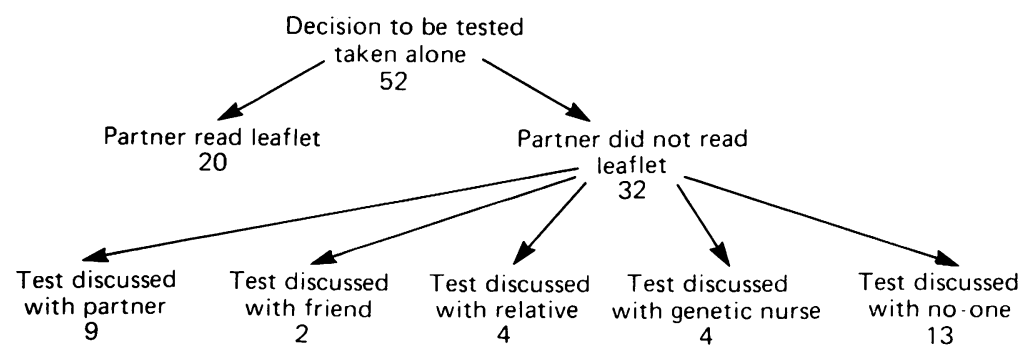

Analysis of discussion with significant others, among those women who took the decision alone, to enter the CF screening trial. perceived change of self image in those identified as gene carriers. ${ }^{11}$

A prerequisite of prenatal CF carrier screening is cooperation of the male partner. Most partners $(63 \%)$ read the prescreening leaflet and were involved in the test decision (table 2). The partners of all 10 women who declined the test had read the leaflet. Although two of these women took the decision to decline the test alone, they had discussed it with their partner. Of those who found the leaflet difficult to understand, five partners read the leaflet and three also found it difficult to understand. Nevertheless, they were party to the decision to be screened. All partners of those women who felt they did not understand the purpose of CF screening were involved in discussion and decision making, although only one had read the leaflet.

Twenty-one respondents (14\%) who entered the trial discussed taking the test with a friend and $18(12 \%)$ with a relative. Only five respondents had talked with their GP and one woman to her health visitor. Fifty-two women made the decision to take the test alone. A concern which emerged from this study is that $13(9 \%)$ women took the decision to be tested without discussing the test with either their partner or other significant person (figure). Evidence suggests that social network and social support play an important role in health, individual decision making, and behaviour. Moreover, social support is known to serve a protective function by moderating the impact of stress and facilitating coping. ${ }^{12}$ It could be hypothesised that good social support will contribute positively to a woman's adjustment to being a CF carrier. It has been pointed out that the whole area of psychosocial support has been neglected in the provision of antenatal care and that careful assessment of a woman's social support system should be an integral part of the antenatal booking procedure. ${ }^{13}$ Identifying vulnerable women in advance of any screening test is important. This study suggests that around $10 \%$ of women booking at the antenatal clinic may have reduced perceived or received social support. Further research is needed to substantiate this.

Those women who decided to accept the CF screening test did so, in $76 \%$ of cases, to avoid the birth of a child with CF. In addition, $71 \%$ stated, either with or without saying they wished to avoid having a CF child, that they were interested to know if they carried a single CF gene. In $16 \%$ of cases, the male partner's wishes had been instrumental in their decision to be tested. Those who gave additional reasons for being screened $(7 \%)$ stated they wished to help with research or contribute to a better understanding of the disorder. Commendable though this is, we have subsequently discouraged women from entering the trial for these reasons. A positive test result has far reaching consequences which prospective participants must consider carefully.

A vast majority (99\%) of women who accepted the screening test thought it 'not very likely' or 'most unlikely' that they would be found to be carriers (table 3). Nevertheless, 
$41 \%$ were still 'anxious' or 'slightly apprehensive' about being screened. There was no correlation between difficulty in understanding the leaflet or understanding the purpose of CF screening and feelings of anxiety towards being tested. The one participant who stated the test made her feel anxious had taken the decision to be tested only after discussion with her GP. She did not think it very likely that she would carry a single CF gene. The whole area of anxiety generated by this programme of screening will be explored in greater depth.

"More about people with the disease, effects, lifespan, etc" was a typical request made by the $15 \%$ of respondents who felt that the leaflet should give more information (table 2). However, those who felt the leaflet offered sufficient information frequently commented that, although they would like to know more about the disease, additional information in the leaflet might only serve to confuse. As a compromise we expanded, slightly, the section of the leaflet entitled 'The disease cystic fibrosis' and, as an adjunct, designed a separate leaflet devoted to the disorder. This leaflet is made readily available at the antenatal clinic.

Good quality educational materials can help promote the relationship between the patient and the health care professional as well as enhancing patient knowledge and self care. ${ }^{5}$ Quality of text will be determined by its readability and must, therefore, be written on a level that is appropriate for the target population. Moreover, readability will be influenced by the content, style, layout, colour, and illustrations. It is unrealistic to believe that a leaflet can provide blanket coverage of all aspects of antenatal CF carrier screening. A mixture of information giving, teaching, and counselling is needed. Nevertheless, the delivery of information by leaflet is one very important strategy. We shall, therefore, continue to evaluate the delivery of prescreening information by leaflet for the duration of this trial.

We are grateful to all the antenatal patients who responded to the questionnaire and took time to comment about the CF carrier testing prescreening information leaflet. Copies of the leaflet may be obtained from the authors on request. This work was supported by a grant from the Cystic Fibrosis Research Trust.

1 Shrimpton AE, McIntosh I, Brock DJH. The incidence of different cystic fibrosis mutations in the Scottish population: effects on prenatal diagnosis and genetic counselling. f Med Genet 1991;28:317-21.

2 Brock DJH. Population screening for cystic fibrosis. $\mathrm{Am} \mathcal{J}$ Hum Genet 1990;47:164-5.

3 Marteau TM, Johnston M, Plenicar M, et al. Development of a self-administered questionnaire to measure women's knowledge of prenatal screening and diagnostic tests. $\mathcal{f}$ Psychosom Res 1988;32:403-8.

4 Wilfond BS, Fost N. The cystic fibrosis gene: medical and social implications for heterozygote detection. $\mathcal{J} A M A$ 1990;263:2777-83.

5 Farrell-Miller P, Gentry P. How effective are your patient education materials? Guidelines for developing and evaluating written educational materials. Diabetes Educ 1989;15:418-22.

6 Bhopal RS, Gilmour WH, Fallon CW, et al. Evaluation of a practice information leaflet. Fam Pract 1990;7:132-7.

7 Merrill CR, Knox AM. Patient attitudes to body computed tomography: use of an information leaflet. Clin Radiol 1986;37:537-8.

8 Council of Europe Official Statement. Genetic screening: European proposals. IME Bull August 1989:10-11.

9 Houston MJR, Field PA. Practices and policies in the initiation of breastfeeding. $\mathcal{f}$ Obstet Gynecol Neonatal Nurs 1988;17:418-24.

10 Faden RR, Chwalow AJ, Orel-Crosby E, et al. What participants understand about a maternal serum alphafetoprotein screening program. Am $\mathcal{f}$ Public Health 1985;75:1381-4.

11 Clow CL, Scriver CR. Knowledge about and attitudes toward genetic screening among high-school students: the Tay-Sachs experience. Pediatrics 1977;59:86-91.

12 Cobb S. Social support as a moderator of life stress. Psychosom Med 1976;38:300-14.

13 Field PA. Effectiveness and efficacy of antenatal care. Midwifery 1990;6:215-23. 